\title{
Application and development of the servo controller based on PXI
}

\author{
Xinghong $\mathrm{Li}^{1, \text { a }}$,Qian Xiang ${ }^{2, b}$ \\ 1,2 The Engineering \& Technical college of Chengdu University of echnology,Leshan,Sichuan,China \\ a545178279@qq.com, b1139916444@qq.com
}

Keywords: subsystems;PXI bus;integration;DMA

Abstract:O-E theodolite is consisted of the subsystems. At present, each subsystem is emplaced separately and connected by cables, which leads to a comparably enormous volume. Therefore, the application of PXI bus technology is optimum that allows inserting every subsystem in a PXI chassis thus largely reduce the overall system volume and improve its integration. This card supports several transmitting modes, including single, burst and DMA.

\section{Introduction}

The photoelectric theodolite is an optical measuring instrument used to measure the trajectory data and flight state in the shooting range, it is also the basic equipment to calibrate the radio measurement and control equipment. Compared with the radio measurement equipment, it has the advantages of high precision, strong visual (can record the target image), and no interference from the ground clutter.

The photoelectric theodolite is consisted of servo control subsystem, data communication subsystem, measurement subsystem, main control TV and many other subsystems, each subsystem is using their own chassis package currently, and then use the integrated conductive ring all the components of the system, resulting in huge volume system. Based on this, this paper uses standard PXI chassis to encapsulate all the components of the subsystem, means in the engineering application, the servo control subsystem, data communication subsystem, the system measurement subsystem, TV are inserted in a PXI case. In order to reduce the volume of the system and improve the integration of the system, then an open control platform is established. If all the subsystems are integrated in a PXI chassis, a universal electronic control platform based on PXI bus can be built.

Based on the PXI bus on the development of servo controller based on PXI bus in this paper, to replace the current servo control system based on PC104 bus universal, so as to realize each sub-system in a PXI case.

\section{Servo controller hardware design}

\section{The principle and composition of the general servo controller}

Photoelectric theodolite with automatic tracking, number of lead tracking and manual tracking, etc., can be selected by the host or panel buttons to track. When the automatic tracking mode is used, the amount of miss distance and the encoder value are sampled regularly, and the relevant control algorithm is adopted to drive the azimuth and pitch motor, so as to make the target within the range of the optical field. When using the manual tracking mode, manipulator through telescope to detect errors, manipulation of single shot target tracking; tracking mode in citation number, computer or other equipment (such as radar) give speed and position of the target, guiding theodolite tracking target. Based on this, the servo controller usually has the following function modules:

(1) control of the core module: the implementation of servo control procedures to track the 
target, coordinate the operation of each module;

(2) IO interface module: read the status of the operation panel, display the current control state;

(3) asynchronous serial interface module: asynchronous serial communication with measuring TV, encoder and master computer;

(4) AD conversion module: read the analog voltage value of single pole.

\section{servo controller based on PXI bus}

Based on the current PC104 servo controller, this paper develops a servo controller based on PXI bus. The controller consists of the following modules:

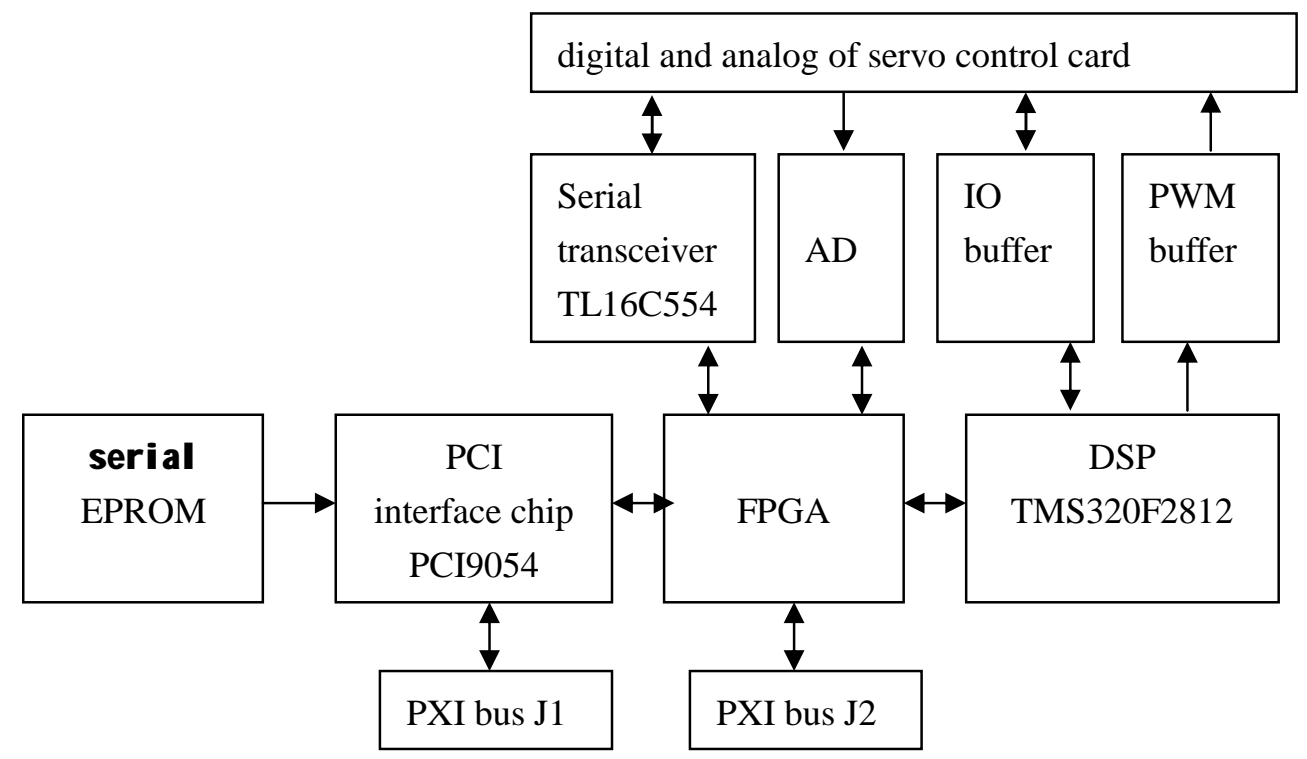

Fig. 1 hardware principle diagram of PXI bus servo control card

(1) control core and IO module

Using DSP (TMS320F2812) as the control core, in the internal operation of the servo control program, generate PWM signal to control the azimuth and elevation of the motor for tracking turntable drive of photoelectric theodolite, the target is always within the optical field of view. At the same time, the IO resources of DSP are used to sample and output IO signals (such as reading the system operation panel status information and displaying the current state information, etc.).

(2) serial communication module

Because the pitch and azimuth angle encoders are usually installed on the machine, it is connected with the servo controller by RS422 serial communication. Using SN74175/SN74174 to achieve the conversion between RS422 differential signal and TTL signal, using asynchronous serial communication chip TL16C554 to achieve the conversion between serial data and parallel data. When communicating with other subsystems, TL16C554 adopts interrupt mode when receiving data, and when the data is sent, the query mode is used.

(3) AD module

In manual tracking mode, it need to use a single rod to track the target. Because the output signal of single pole is analog signal, AD7864 is used to carry out the conversion from analog to digital.

(4) PXI interface module

The PCI interface is realized by using the special PCI interface chip (PCI9054). The host through the PCI bus to send or receive information from the servo controller number of miss distance information, at the same time, local bus and PXI trigger bus into FPGA, in order to realize the servo control subsystem, data communication subsystem, time sharing subsystem, measuring television subsystems provide hardware support to communicate with each other. 


\section{the block diagram of FPGA design}

According to the structure and function of the servo control card, modular design, FPGA design of modular decomposition, such as FPGA need to deal with the PCI local bus, also need to provide TL16C554 and AD7864 chip select and read and write signal etc..

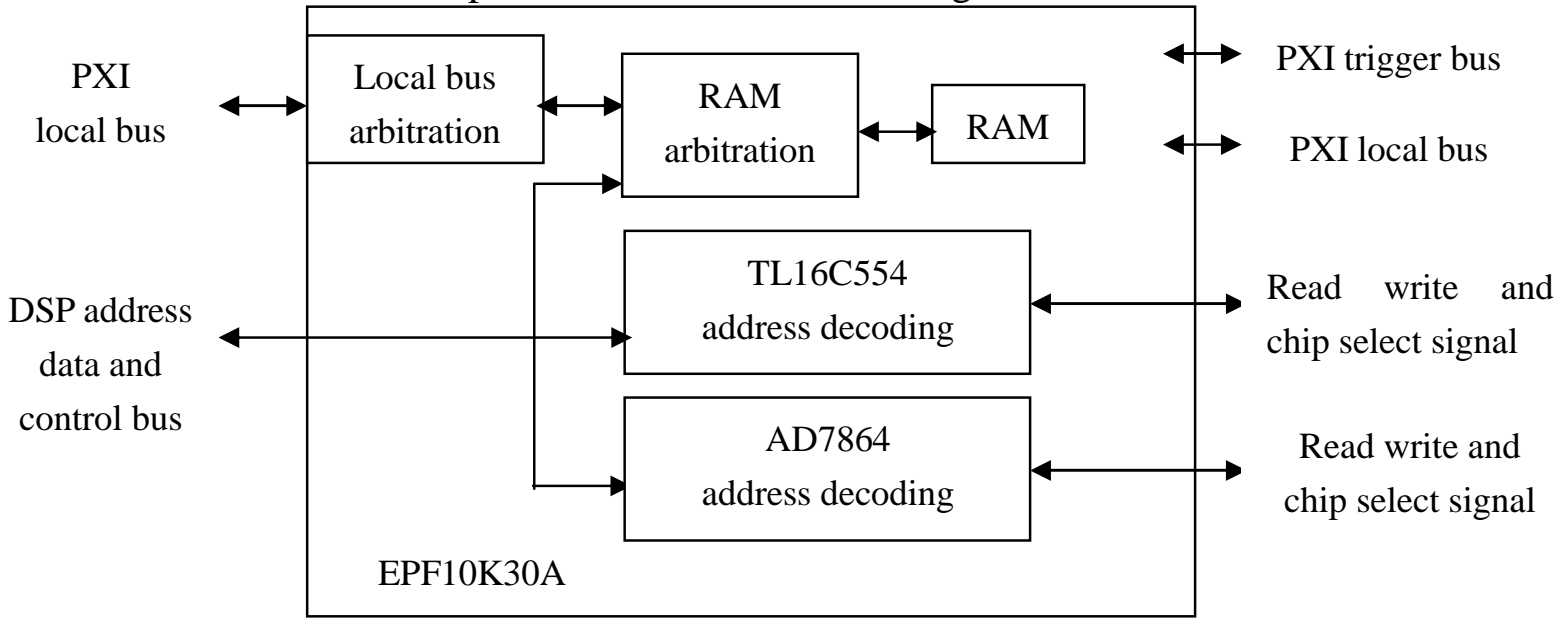

Fig. 2 block diagram of FPGA design

\section{module design of serial transceiver}

In the existing optoelectronic devices, the communication between each subsystem is based on RS422 serial communication. Servo control card communicate with the encoder module, it is necessary to provide a serial communication interface, using TL16C554 to complete the conversion between parallel data and serial data. Between the servo control card and the external serial communication, TL16C554 adopts the interrupt mode when receive, while adopts the inquiry mode when send out.

\section{the driver design of PXI bus servo controller}

Previously, the general hardware equipment and computer related are standard equipment, the manufacturer or the specialized program developers provided the driver and package, users only need to understand Windows API which connected to the drivers, without the need to know its internal operation mechanism. However, if the hardware is a non-standard device, it is necessary to design the device driver for the Windows environment in order to complete the application's access to the hardware. In the design and use of PCI devices, often access the system resources in the software, so only the preparation of device drivers to achieve full access to the PCI bus device.

On the basis of the kernel structure of Window2000 and the WDM driver model, the WDM driver of the servo control card is developed by using the popular DriverStdio tools.

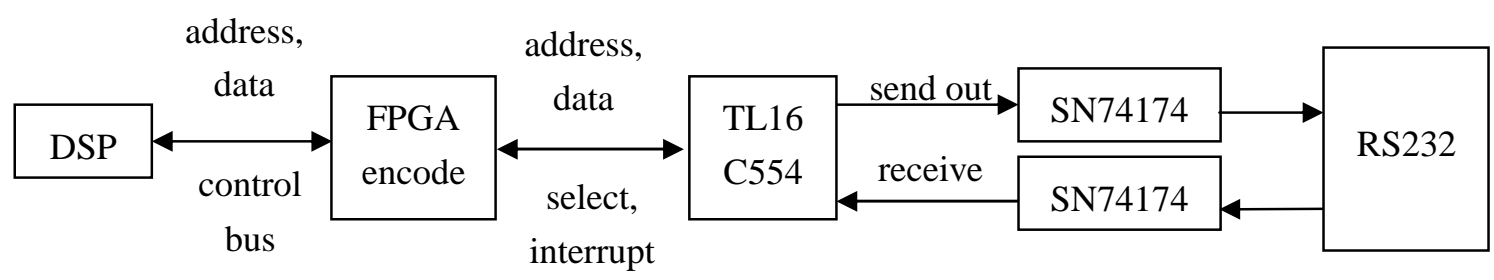

Fig. 3 Schematic diagram of serial communication

\section{DMA programming}

Because the control DMA transmission without CPU, after settings related parameters (such as memory on both sides of the starting address and the transmission length, etc.), between two memories (or between host and PCI device) can directly transfer data, so it is widely used in 
high-speed data transmission. In this paper, the PCI device is PC19054, it comes with DMA controller, by means of setting the PCI9054 related registers to select the relevant DMA transmission mode.

PCI9054 supports two DMA transfer modes: Block mode and Scatter/Gather mode. Taking into account the actual situation, the host application uses a common buffer DMA to create a buffer, while the PCI device (servo control card) uses Block transmission mode.

\section{the procedure of DMA transmission}

The application calls the driver DMA peripheral device for write operation as an example to illustrate the transmission process of DMA, the servo control card of PCI9054 works with slave mode, therefore requires the host to initiate the read and write operation.

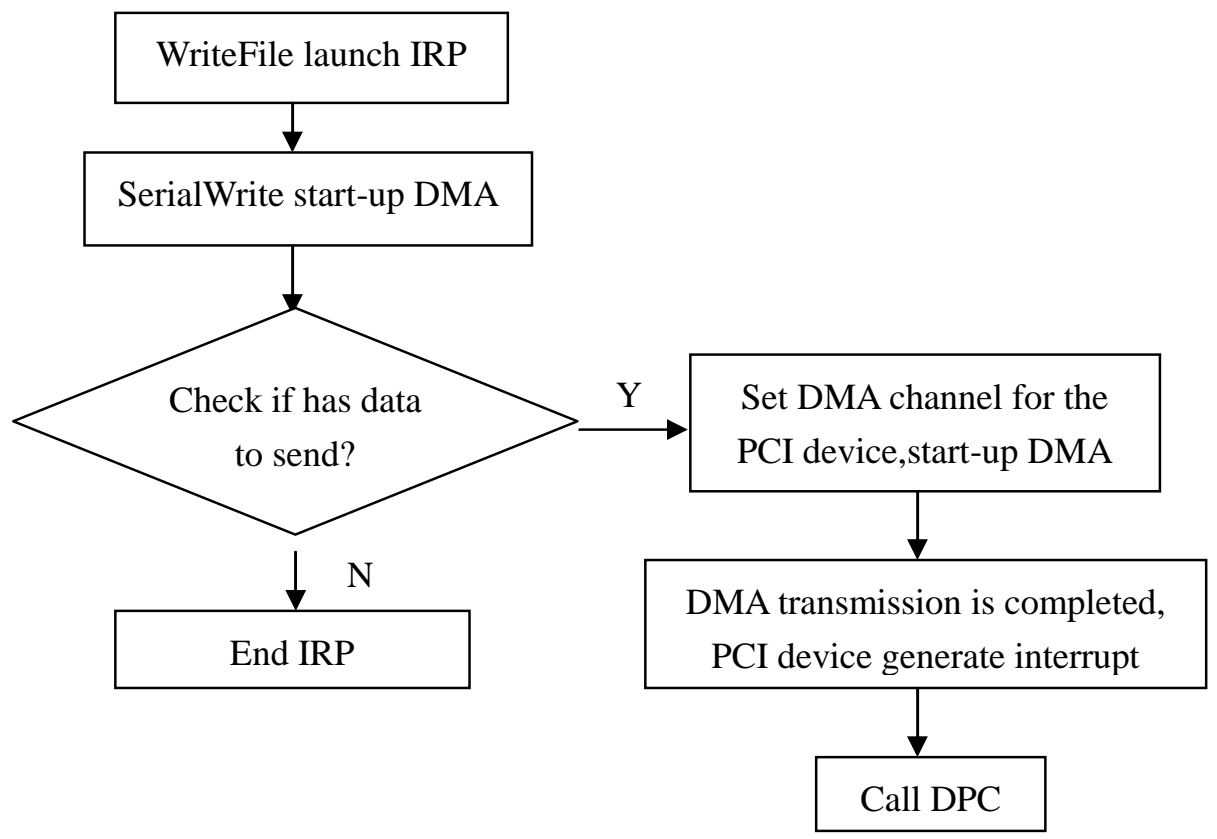

Fig. 4 DMA transmission flow

\section{communication between driver and application}

In Windows, the process between implementation of the application and WDM communication is: application CreatFile function is used to open the device, then use the DeviceIoControl and WDM driver for communication, also can use the ReadFile to read data from WDM or use WriteFile to write data to the WDM. When the application exits, use CloseHandle to turn off the device.

Due to the servo control card in the process of work, DSP and the host exchange data through the embedded RAM of FPGA, this RAM is shared by DSP and host. In order to achieve shared RAM, interrupt mode is adopted. After DSP writes the specified length of data to the shared RAM, it sends an interrupt to the host, telling the host that the data is ready. When the host receives the interrupt signal, the data in the RAM is read out by the PCI bus, so the host receives the data sent by the DSP. Similarly, the host can also write data to the shared RAM through the PCI bus, and then send an interrupt signal to the DSP. DSP receives the interrupt signal sent by the host, the data will be read out in the RAM, so that the DSP and the host achieve the exchange data.

As can be seen from the host application in the exchange of data with the DSP process, the driver needs to capture the interrupt occurs, and notify the application to read data. This requires communication between the driver and the application. In the process of developing the servo control card, the communication between the application program and the driver is carried out by event notification, the specific steps of its programming are as follows: 
(1) the event object is created in the application, and the event object through the DeviceIoControl function to send to the driver, at the same time open up related threads in the application program, for receiving the event messages sended by driver program.

(2) when the driver receives an event notification, it creates a KEvent object that corresponds to the event object created by the application.

(3) after the driver receives the interrupt from the hardware, it sets the event as a signal state in the interrupt service program, which is called m_pEventRead.Set. After the event receive thread DmaReadProc () of the application receives the event message, stops waiting and starts running down. After performing related operations(such as reading data from the device), wait for the driver to send event notifications again. At the same time, the driver continues to send an event message to the application after receiving the interrupt signal. This realizes the communication between the driver and the application.

\section{the test of data transfer rate between the host and the servo control card}

Based on the normal communication between the host and the servo control card, it is necessary to measure the data transfer rate between them. At present, there are two methods, one is software measurement, the other is hardware measurement.

\section{software measurement}

VC is usually used in the process of writing the timer, have to call the API function SetTimer to establish a timer, it can reach the millisecond timing accuracy. However, the data transmission rate of PCI bus is fast, in order to measure the data transfer rate between the host computer and the servo control card, the timing accuracy of MS can not meet the requirements, so this paper uses hardware method to measure.

\section{hardware measurement}

Hardware measurement refers to the application program send or receive data to servo control card, using oscilloscope to observe the change cycle from BLAST\# and ADS\# signal of PCI9054, can get the transmission time of each group of data, which can calculate the data transfer rate between the host and servo control card.

\section{test results of the data transfer rate}

In the case of a 8 bit data bus, which are divided into two kinds of data transmission between the host and the servo control card for rate measurement, one is the transmitting data rate of host sent data to a servo control card, the other is the transmitting data rate of host receive data from servo control card.

Host applications send data to the servo control card constantly, the use of hardware measurement method to measure the transmission rate of data in various transmission modes, measured as shown in table 1:

Table 1 data transfer rate from host to servo control card

\begin{tabular}{|l|l|c|c|c|}
\hline \multicolumn{2}{|c|}{ Transmission mode } & byte count (Byte) & time(us) & data transfer rate (Mbyte/s) \\
\hline \multicolumn{2}{|l|}{ Single cycle write in slave mode } & 1024 & 240 & 4.27 \\
\hline \multicolumn{2}{|c|}{ Burst write in slave mode } & 1024 & 145 & 7.06 \\
\hline \multirow{3}{*}{\begin{tabular}{l}
\multirow{2}{*}{$\begin{array}{l}\text { DMA } \\
\text { mode }\end{array}$} \\
\cline { 2 - 5 }
\end{tabular}} & single cycle write & 1024 & 115 & 8.90 \\
\cline { 2 - 5 } & Burst write & 1024 & 60 & 17.07 \\
\cline { 2 - 5 } & Infinite burst (BTERM\#mode) & 1024 & 39 & 26.3 \\
\hline
\end{tabular}

Host applications read data from the servo control card constantly, the use of hardware measurement method to measure the transmission rate of data in various transmission modes, 
measured as shown in table 2:

Table 2 data transfer rate of the host read servo control card data

\begin{tabular}{|c|c|c|c|c|}
\hline \multicolumn{2}{|c|}{ Transmission mode } & byte count (Byte) & time(us) & data transfer rate (Mbyte/s) \\
\hline \multicolumn{2}{|c|}{ Single cycle read in slave mode } & 1024 & 925 & 1.11 \\
\hline \multicolumn{2}{|c|}{ Burst read in slave mode } & 1024 & 260 & 3.94 \\
\hline \multirow{3}{*}{$\begin{array}{l}\text { DMA } \\
\text { mode }\end{array}$} & single cycle read & 1024 & 110 & 9.31 \\
\hline & Burst read & 1024 & 58 & 17.65 \\
\hline & Infinite burst (BTERM\#mode) & 1024 & 39 & 25.26 \\
\hline
\end{tabular}

From the table, in the case of the 8 bit data bus, speed up to 26.3Mbyte/s at BTERM\# mode. If the local data bus uses 32 bits, the data transmission rate can reach $26.3 * 4=105.2 \mathrm{Mbyte} / \mathrm{s}$, close to the PCI limit transmission rate, can meet most of the high speed data transmission needs.

\section{concluding remarks}

In this paper, based on the relevant technology of PXI bus, the servo controller based on PXI bus is developed, which realizes the integration of each subsystem in the same PXI chassis. Host can communicate with DSP through RAM created by FPGA, design PCI9054 local bus controller in FPGA, and embedded single port RAM in the FPGA. When PCI9054 works in the slave mode, PXI servo controller supports single cycle, burst and DMA transmission mode, and the use of DMA infinite burst transmission mode, in the case of a 8 bit data bus, data transmission rate close to the transmission rate of PCI. By using Visual $\mathrm{C}++$ to write the application program, the experimental platform of PCI bus is established. In this platform, users can complete a variety of transmission experiments, including single cycle, burst and DMA mode. To improve the system integration, modularization and standardization of each subsystem will be the future development direction of photoelectric measurement equipment, so the PXI bus technology will be widely used in the photoelectric measurement equipment.

\section{Reference:}

[1] Wuan River and other.Windows 2000/XP WDM device driver development [M]. Electronic Industry Press.2005.05

[2] Zhou Min, based on PXI bus data acquisition system interrupt handling mechanism and DMA programming [J], measurement and control technology, 2005, 9

[3] Wu Guoqing, modern measurement and control technology and application [M], publishing house of electronics industry, 2007, 8

[4] Chen Kang, Wang Xudong, based on the PXI bus field equipment universal detection system design [J], measurement and control technology, 2008, 7-191 